\title{
Infection with hepatitis B and C virus in Europe: a systematic review of prevalence and cost-effectiveness of screening
}

\author{
Susan JM Hahné ${ }^{*}$, Irene K Veldhuijzen², Lucas Wiessing ${ }^{4}$, Tek-Ang Lim³ ${ }^{3}$ Mika Salminen ${ }^{3}$ and Marita van de Laar ${ }^{3}$
}

\begin{abstract}
Background: Treatment for chronic hepatitis B virus (HBV) and hepatitis C virus (HCV) infection is improving but not benefiting individuals unaware to be infected. To inform screening policies we assessed (1) the hepatitis B surface antigen (HBsAg) and anti-hepatitis C virus antibody (anti-HCV-Ab) prevalence for 34 European countries; and (2) the cost-effectiveness of screening for chronic HBV and HCV infection.

Methods: We searched peer-reviewed literature for data on HBsAg and anti-HCV-Ab prevalence and cost-effectiveness of screening of the general population and five subgroups, and used data for people who inject drugs (PWID) and blood donors from two European organizations. Of 1759 and 468 papers found in the prevalence and cost-effectiveness searches respectively, we included 124 and 29 papers after assessing their quality. We used decision rules to calculate weighted prevalence estimates by country.

Results: The HBsAg and anti-HCV-Ab prevalence in the general population ranged from $0.1 \%-5.6 \%$ and $0.4 \%-5.2 \%$ respectively, by country. For PWID, men who have sex with men and migrants, the prevalence of HBsAg and anti-HCV-Ab was higher than the prevalence in the general population in all but 3 countries. There is evidence that HCV screening of PWID and HBsAg screening of pregnant women and migrants is cost-effective.

Conclusion: The prevalence of chronic HBV and HCV infection varies widely between European countries. Anti-HCV-Ab screening of PWID and HBsAg screening of pregnant women and migrants have European public health priority. Cost-effectiveness analyses may need to take effect of antiviral treatment on preventing HBV and HCV transmission into account.
\end{abstract}

Keywords: Hepatitis B virus, Hepatitis C virus, Europe, Prevalence, HBsAg, Anti-HCV-Ab, Cost-effectiveness analyses

\section{Background}

Hepatitis B and C virus (HBV and HCV) infect the liver and can lead to a broad spectrum of disease outcomes. Between $15 \%$ and $40 \%$ of those chronically infected with $\mathrm{HBV}$ or $\mathrm{HCV}$ will in their lifetimes develop serious liver disease due to cirrhosis and/or hepatocellular cancer (HCC) [1,2]. People with chronic infection with HBV or $\mathrm{HCV}$ can remain infectious to others. Both HBV and $\mathrm{HCV}$ are widely present with broad variation in prevalence by country [3]. Worldwide, between 350 to 400 million people are infected with $\mathrm{HBV}$, accounting for 1

\footnotetext{
* Correspondence: Susan.Hahne@rivm.nl

${ }^{1}$ Centre for Infectious Disease Control, National Institute for Public Health and the Environment (RIVM), PO Box 1, 3720 BA Bilthoven, The Netherlands Full list of author information is available at the end of the article
}

million deaths per year [4,5]. Between 130 and 170 million people are infected with $\mathrm{HCV}$, causing over 350,000 deaths per year [6].

A safe and effective vaccine for HBV has been available since 1982, whereas no vaccine for HCV exists [7]. Treatment options are advancing rapidly, and several new antiviral drugs have become available in the past decade. Evidence is accumulating that these therapies provide a cost-effective means to reduce the morbidity and mortality associated with chronic infection with HBV and HCV [8-10]. European treatment guidelines for chronic HBV and HCV infection are available [11,12]. In addition to improving the outcome of chronic hepatitis, antiviral treatment is likely to reduce transmission by reducing the viral load and therefore infectivity of chronic

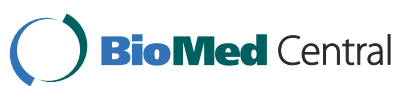


carriers, similar to what has been documented for HIV [13-15]. For HBV, vaccination of susceptible contacts of identified carriers can prevent new infections. Nonpharmaceutical interventions, such as the advice to limit alcohol intake and cease smoking, can improve outcomes for people living with chronic viral hepatitis $[16,17]$.

Since the acquisition of HBV and HCV is often asymptomatic or subclinical, and sequelae take several decades to develop, between $40 \%$ and $80 \%$ of people with chronic hepatitis are unaware of their infection [18-25]. Therefore, screening programmes for chronic HBV and $\mathrm{HCV}$ infection have the potential to contribute considerably to primary and secondary prevention of these infections. However, existing $\mathrm{HBV}$ and $\mathrm{HCV}$ screening programmes in Europe stem from an era when treatment options for chronic viral hepatitis were limited. Hence they are mainly aimed at primary prevention, targeting blood donors, pregnant women, and behavioral high-risk groups [26]. Now that secondary prevention of HBV and $\mathrm{HCV}$ is possible, there is an urgent need to identify chronic carriers who may benefit from treatment.

For policy development in this area data on the size and characteristics of the population with chronic hepatitis and the evidence for cost-effectiveness of screening are needed. The most recent HBsAg prevalence review including data on European countries was from 2004, and reported findings from only 11 European countries [27]. For $\mathrm{HCV}$, a review of the burden of disease in Europe was published in 2009 [28]. In this review, however expert opinion was a main source of data, which makes the validity of conclusions difficult to ascertain. Esteban reviewed the HCV prevalence in Europe in 2008 [29], but studies on blood donors were included as estimates for the general population. Regarding the cost-effectiveness of screening for $\mathrm{HCV}$, an earlier review included studies published up to March 2007 [30]. It concluded that HCV screening of both former and current PWID was cost-effective. Systematic reviews of cost-effectiveness of screening for $\mathrm{HBV}$ infections have not been published.

To address the missing information we performed a systematic literature review of the prevalence of hepatitis $\mathrm{C}$ virus antibodies (anti-HCV-Ab) and hepatitis B surface antigen (HBsAg) in the general population and five population subgroups (pregnant women, first-time blood donors, people who inject drugs [PWID], men who have sex with men [MSM], and migrants) for 34 European countries. $^{\text {a } W e ~ s u b s e q u e n t l y ~ u s e d ~ o u r ~ p r e v a l e n c e ~ e s t i-~}$ mates to assess the total number of people living with chronic HBV and HCV infection by country. To further build the evidence base for secondary prevention we also performed a systematic review of the cost-effectiveness of HBsAg and anti-HCV screening of the general population and population sub-groups.

\section{Methods}

\section{Hepatitis B and C prevalence}

To find studies that describe prevalence of $\mathrm{HBsAg}$ and anti-HCV-Ab (the serological markers used as proxies for chronic infection in this study) we searched Medline, Embase, and SciSearch for English-language, peer-reviewed literature published between 1 January 2000 and 27 July 2009. Reference lists of included studies were hand searched. Studies were eligible for inclusion in the review if they reported the anti-HCV-Ab and/or HBsAg prevalence in the 34 countries included in our review, in the general population or among pregnant women, first-time blood donors, MSM, or migrants. Studies that reported on children only were not included. We only used the most recent estimate when more than 1 regional estimate was available based on studies performed 5 or more years apart. When several estimates were available for a specific country, an average weighted by study size was calculated. For firsttime blood donors, we used data from a report for the Council of Europe in addition to data from the published literature [31]. Anti-HCV-Ab and HBsAg prevalence estimates among PWID were obtained from 2 sources: the European Monitoring Centre for Drugs and Drug Addiction (EMCDDA) [32] and from a recent review on HBV and HCV prevalence among PWID [33]. The source with the most recent national prevalence estimate was used. We excluded estimates from studies performed before 2000, with fewer than 50 participants, and where injecting drug use status was unknown.

\section{Costs effectiveness of screening for hepatitis B and C}

For the systematic literature review of the evidence for cost-effectiveness of screening for chronic HBV and/or $\mathrm{HCV}$ infection we searched Medline, Scopus, and the NHS Economic Evaluation Database (EED) for studies published in the English-language, peer-reviewed literature between 1 January 2000 and 31 December 2012. Studies reporting only on screening of transfusion recipients and/or of patients treated by infected health care workers ('look-back studies') were excluded. Studies were only eligible when reporting estimated costs per additional chronic infection identified and/or costs per life year (LY) gained (quality or disability adjusted). Cost estimates were converted into 2010 Euros using information from Eurostat and OECD [34,35].

\section{Data extraction}

For both systematic literature searches, data were extracted using a data-extraction form by two authors (SH and IV). For the prevalence search, the form included year, country population of the study, the sampling method, laboratory test used, participation rate, number of participants, and HBsAg and anti-HCV-Ab results. For the 
cost-effectiveness search, the form included year and country of study, target population for screening, screening scenario, type of model used, outcome measure(s) used, monetary value and year, discounting percentage (costs/effects), results, and conclusions. The quality of the prevalence studies was assessed by reviewing the representativeness of sampling (eg, random vs convenience sampling) and, for the general population, whether estimates were standardized by age and sex.

Prevalence estimates were summarized by country. When multiple general population prevalence estimates were available for one country, we used the estimate that was most representative for the entire country regarding demographic coverage. In case multiple representative general population prevalence estimates for one country were found, the average prevalence was calculated weighted by study size. Where estimates for 3 or more regions in a country were available, regional estimates were presented only when the difference between regions was more than $0.5 \%$. Countries were grouped into low, intermediate, and high $\mathrm{HBsAg}$ and anti-HCV-Ab prevalence using cut offs of $\leq 1 \%,>1 \%$ to $\leq 2 \%$, and $>2 \%$. On the basis of prevalence estimates in the general population for infection with HBV and HCV and 2009 population size [36], we estimated the total number of people in that country who would likely test positive for HBsAg or anti-HCV-Ab. Search terms used for both searches are available in Additional file 1. The methods of our systematic literature reviews and their reporting are consistent with those recommended by the PRISMA statement and specified in advance in a protocol that is available from the corresponding author on request [37].

\section{Results}

\section{Seroprevalence of hepatitis B and C}

The search for data on the HBsAg and/or anti-HCV-Ab prevalence in the general population and 5 subgroups identified 1759 citations, from which the full-text publication of 236 (13\%) was retrieved. From the reference lists of included studies, an additional 8 potentially relevant citations were identified. After review of the full text of these 244 papers, 53 publications were considered not relevant. Furthermore, 67 publications on PWID were excluded, since prevalence estimates from PWID were obtained from the EMCDDA and a recent literature review [33]. Finally, 124 publications were included in the review of prevalence data, with 81 publications used for the prevalence estimate for the general population or population subgroups. A flow diagram depicting the inclusion of studies is available in the Additional file 2.

\section{General population}

HBsAg general population prevalence estimates were found for 13 of the 34 countries in our review, ranging from $0.1 \%$ to $5.6 \%$ by country (Figure $1 \mathrm{a}$, Table 1 ). The estimated number of people with chronic HBV infection ranged from $3,718,889$ in Turkey to 4,466 in Ireland (Table 1). Prevalence estimates of anti-HCV-Ab in the general population were found for 13 of the 34 countries in our review, ranging from $0.4 \%$ to $5.2 \%$ by country (Figure 1b, Table 2). The estimated number of people who were anti-HCV-Ab positive ranged from 3,122,779 in Italy to 37,025 in Sweden (Table 2). For only a minority of countries (9/34) information was available on both estimates. Countries in the north-western part of Europe had a low prevalence for both infections whilst those in the south and south-east had an intermediate to high prevalence (Additional file 3: Figure S2).

\section{Blood donors}

HBsAg prevalence estimates for first-time blood donors were found for 24 countries, ranging from $0.0 \%$ to $5.2 \%$ (Additional file 3: Figure S3.1a, Additional file 4: Table S3.1.a). Anti-HCV-Ab prevalence estimates for firsttime blood donors were available for 23 countries, ranging from $0.02 \%$ to $3.3 \%$ (Additional file 3: Figure S3.1b, Additional file 4: Table S3.1.b). The prevalence of HBsAg and anti-HCV-Ab in first-time blood donors was on average respectively 3 and 4 times lower than the corresponding prevalence for the general population in countries that had both estimates available (12 countries for HBsAg and 11 for anti-HCV-Ab).

\section{Pregnant women}

Estimates of antenatal HBsAg prevalence were found for 11 countries, ranging from $0.1 \%$ to $4.4 \%$ (Additional file 3: Figure S3.2a, Additional file 4: Table S3.2.a). Estimates of antenatal anti-HCV-Ab prevalence were found for 6 countries, ranging from $0 \%$ to $1.7 \%$ (Additional file 3: Figure S3.2b, Additional file 4: Table S3.2.b). The antenatal HBsAg prevalence was on average 3 times higher than the general population prevalence in 6 of the 7 countries that had both estimates available. The country where it was lower was Spain (based on regional data from Catalonia), likely reflecting the effect of the HBV vaccination programme for adolescents. In Italy and the United Kingdom, the antenatal anti-HCV-Ab prevalence was lower than the general population prevalence. In Germany and Greece, it was higher.

\section{PWID}

An estimate of HBsAg prevalence in PWID was available for 21 of the 34 countries in this review, ranging from $0 \%$ to $21.3 \%$ (Additional file 3: Figure S3.3a, Additional file 4: Table S3.3.a). An estimate of anti-HCV-Ab prevalence in PWID was available for 29 of the 34 countries, ranging from $5.3 \%$ to $90 \%$ (Additional file 3: Figure S3.3b, Additional file 4: Table S3.3.b). The HBsAg prevalence in 
PWID was on average 9 times higher than that in the general population (in 6 of the 8 countries that had both estimates available). In Romania and Ireland, the general population HBsAg estimate was higher. The estimate of anti-HCV-Ab prevalence in PWID was on average 47 times higher than that in the general population (in 13 countries that had both estimates available).

\section{Migrants}

Estimates of HBsAg prevalence in migrants were found for 5 countries. The HBsAg prevalence in migrants ranged from 1.0\% to 15.4\% (Additional file 4: Table S3.4.a). Estimates of anti-HCV-Ab prevalence in migrants were found for 5 countries, ranging from $0 \%$ to $23.4 \%$ (Additional file 4 : Table S3.4.b). The estimate of HBsAg and anti-HCV-Ab prevalence in migrants was on average respectively 6 and 2 times higher than that in the general population in all countries that had both estimates available (4 countries for $\mathrm{HBsAg}$ and 4 for anti-HCV-Ab), except for Italy, where the estimate of anti-HCV-Ab prevalence in migrants was lower than that in the general population.

\section{MSM}

Estimates of HBsAg prevalence for MSM were found for 3 countries, ranging from $<1 \%$ to $4 \%$ [38-41]. Estimates of anti-HCV-Ab prevalence among MSM were available for 3 countries, ranging from $0.07 \%$ to $2.9 \%$ [41-43]. The $\mathrm{HBsAg}$ and anti-HCV-Ab prevalence in MSM was on average respectively 22 and 3 times higher than that for the general population in all countries that had both estimates available (2 countries for $\mathrm{HBsAg}$ and 1 for anti$\mathrm{HCV}-\mathrm{Ab})$.

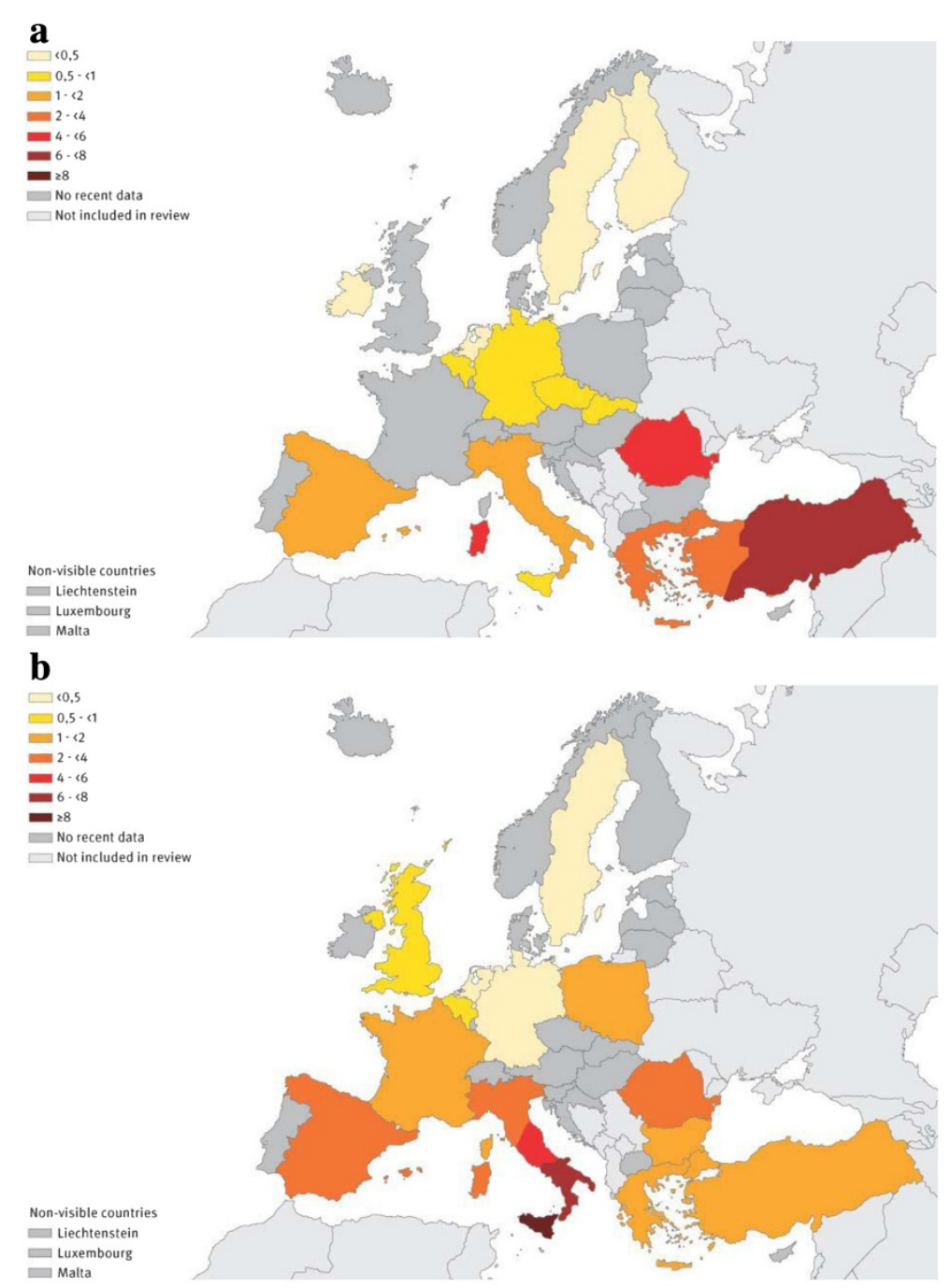

Figure 1 Hepatitis B and hepatitis C prevalence (\%) in the general population by country, Europe, 2000-2009. a. Hepatitis B surface antigen (HBsAg) prevalence (\%). b. Hepatitis C (anti-HCV-antibody) prevalence (\%). 
Table 1 Estimates of general population HBsAg prevalence, and number of HBsAg positive people in the general population, by country, Europe, $2000-2009$

\begin{tabular}{|c|c|c|c|c|c|c|c|c|c|c|}
\hline Country* (Reference) & Period & Area & Region & Sampling & $\mathrm{N}$ & $\%$ & $(95 \% \mathrm{Cl})^{* *}$ & Remarks & Population size [36] & $\begin{array}{l}\text { Number of HBsAg-positive } \\
\text { inhabitants }\end{array}$ \\
\hline Belgium [91] & 2003 & regional & Flanders & random & 1.834 & 0,7 & $(0,5-0,8) \&$ & Oral fluid & 10.754 .528 & 75.282 \\
\hline Czech Republic [92] & 2001 & nationwide & - & random & 2.658 & 0,6 & $(0,3-1,0) \$$ & Standardized & 10.467 .542 & 62.805 \\
\hline Finland [93] & 1997-1998 & nationwide & - & residual & 3.083 & 0,2 & $(0,1-0,4) \$$ & & 5.326 .314 & 10.653 \\
\hline Germany [94] & 1993-1996 & nationwide & - & random & 5.305 & 0,6 & $(0,4-0,8) \&$ & & 82.050 .000 & 492.300 \\
\hline Germany [95] & 1998 & nationwide & - & random & 6.748 & 0,6 & $(0,4-0,8) \&$ & & & \\
\hline Greece [96] & 1997-1998 & regional & Peloponnesos & random & 1.500 & 2,1 & $(1,5-3,0) \&$ & & 11.257 .285 & 236.403 \\
\hline Ireland [93] & 2003 & nationwide & - & residual & 2.535 & 0,1 & $(0,0-0,3) \$$ & & 4.465 .540 & 4.466 \\
\hline Italy [93] & 1996 & nationwide & - & residual & 3.522 & 0,6 & $(0,4-1,0) \$$ & & 60.053 .442 & 840.748 \\
\hline Italy [74] & 2002 & regional & North & convenience & 956 & 1,0 & $(0,5-1,9) \$$ & & & \\
\hline Italy [97] & 1997 & regional & Central & random & 250 & 1,2 & $(0,3-3,5) \$$ & & & \\
\hline Italy [98] & 1997 & regional & South & random & 488 & 0,2 & $(0,0-3,5) \$$ & & & \\
\hline Italy [99] & $2002-2003$ & regional & South & random & 1.645 & 1,8 & $(0,4-1,2) \&$ & & & \\
\hline Italy [100] & 1994-1994 & regional & Sardinia & convenience & 3.324 & 4,3 & $(3,6-5,1) \$$ & & & \\
\hline Italy [101] & 1999-2000 & regional & Sicily & random & 721 & 0,7 & $(0,2-1,6) \$$ & & & \\
\hline Netherlands [93] & 1995-1996 & nationwide & - & random & 6.750 & 0,1 & $(0,0-0,2) \$$ & & 16.486 .587 & 16.487 \\
\hline Romania [93] & 2002 & nationwide & - & residual & 1.259 & 5,6 & $(4,4-7,0) \$$ & & 21.498 .616 & 1.203 .922 \\
\hline Slovakia [93] & 2002 & nationwide & - & random & 3.569 & 0,6 & $(0,4-0,9) \$$ & & 5.412 .254 & 32.474 \\
\hline Spain [102] & 1996 & regional & Catalonia & random & 2.142 & 1,2 & $(0,7-1,7) \&$ & Standardized & 45.828 .172 & 458.282 \\
\hline Spain [103] & 2002 & regional & Catalonia & random & 2.620 & 0,7 & $(0,4-1,0) \&$ & & & \\
\hline Sweden [104] & 1991-1994 & regional & Malmö & random & 5.533 & 0,2 & $(0,1-0,4) \$$ & & 9.256 .347 & 18.513 \\
\hline Turkey [105] & 2006-2007 & regional & West & random & 2.852 & 2,5 & $(2,0-3,1) \$$ & & 71.517 .100 & 3.718 .889 \\
\hline Turkey [106] & 2002-2004 & regional & Central & convenience & 1.320 & 6,6 & $(5,3-8,1) \$$ & & & \\
\hline Turkey [107] & 1996 & regional & Central & convenience & 571 & 6,7 & $(4,8-9,1) \$$ & & & \\
\hline Turkey [108] & Not reported & regional & Central & random & 1.095 & 5,5 & $(4,2-7,0) \$$ & & & \\
\hline Turkey [109] & 1997-1999 & regional & East & convenience & 400 & 9,0 & $(6,4-12,2) \$$ & 32-year-olds & & \\
\hline Turkey [110] & 2003 & regional & East & random & 2.888 & 7,0 & $(6,1-8,0) \$$ & & & \\
\hline
\end{tabular}

* No estimate of HBsAg general population prevalence was found for Austria, Bulgaria, Croatia, Denmark, Estonia, Former Yugoslav Republic of Macedonia, France, Hungary, Iceland, Latvia, Liechtenstein, Lithuania, Luxembourg, Malta, Norway, Poland, Portugal, Slovenia, Switzerland and the United Kingdom.

** $\mathrm{Cl}$ Confidence interval.

$\& \mathrm{Cl}$ provided in the original paper.

$\$ \mathrm{Cl}$ estimated by the exact method. 
Table 2 Estimates of general population anti-HCV-Ab prevalence, and number of anti-HCV positive people in the general population, by country, Europe, 2000-2009

\begin{tabular}{|c|c|c|c|c|c|c|c|c|c|c|}
\hline Country* (Reference) & Period & Area & Region & Sampling & $\mathbf{N}$ & $\%$ & $95 \% \mathrm{Cl}^{* *}$ & Remarks & Population size [36] & $\begin{array}{l}\text { Number of inhabitants who } \\
\text { are anti-HCV-Ab positive }\end{array}$ \\
\hline Belgium [91] & 2003 & regional & Flanders & random & 1.834 & 0,1 & $(0.1-0.4) \&$ & Oral fluid & 10.754 .528 & 64.527 \\
\hline Belgium [111] & 1993-1994 & regional & Flanders & random & 4.055 & 0,9 & $(0.5-1.1) \&$ & & & \\
\hline Bulgaria [112] & 1999-2000 & regional & South-Central & convenience & 2.211 & 1,3 & $(1.2-1.4) \&$ & Standardized & 7.606 .551 & 98.885 \\
\hline Czech Republic [92] & 2001 & nationwide & - & random & 2.658 & 0,2 & $(0.1-0.4) \$$ & & 10.467 .542 & 20.935 \\
\hline France [113] & 1997 & regional & South & convenience & 11.804 & 1,3 & $(1.1-1.5) \&$ & & 64.351 .000 & 836563 \\
\hline Germany [95] & 1998 & nationwide & - & random & 6.748 & 0,4 & $(0.2-0.5) \&$ & & 82.050 .000 & 328200 \\
\hline Greece [96] & 1997-1998 & regional & Peloponnesos & random & 1.500 & 0,5 & $(0.2-1.1) \&$ & & 11.257 .285 & 112.573 \\
\hline Greece [114] & 1997 & regional & Zakinthos & random & 718 & 1,3 & $(0.6-2.4) \$$ & & & \\
\hline Italy [74] & 2002 & regional & North & convenience & 956 & 2,6 & $(1.7-3.8) \$$ & & & \\
\hline Italy [115] & 1994-1995 & regional & North & convenience & 2.154 & 3,3 & $(2.6-4.1) \&$ & & & \\
\hline Italy [116] & Not reported & regional & North & convenience & 4.820 & 2,4 & $(2.0-2.8) \&$ & & & \\
\hline Italy [117] & Not reported & regional & Central & convenience & 300 & 16,3 & $(12.0-20.6) \&$ & & & \\
\hline Italy [97] & 1997 & regional & Central & random & 250 & 22,4 & $(20.8-24.1) \&$ & & & \\
\hline Italy [98] & Not reported & regional & South & random & 488 & 16,2 & $(13.0-19.8) \$$ & & & \\
\hline Italy [118] & $2000-2002$ & regional & South & convenience & 2.753 & 7,9 & $(6.9-9.0) \$$ & & & \\
\hline Italy [99] & $2002-2003$ & regional & South & random & 1.645 & 6,5 & $(5.3-7.7) \&$ & & & \\
\hline Italy [100] & 1994-1995 & regional & Sardinia & convenience & 3.324 & 3,2 & $(2,6-3,8) \$$ & & & \\
\hline Italy [101] & $1999-2000$ & regional & Sicily & random & 721 & 10,4 & $(8,2-12,9) \$$ & & & \\
\hline Netherlands [41] & 2004 & regional & Amsterdam & random & 1.364 & 0,6 & $(0.1-1.1) \&$ & Standardized & 16.486 .587 & 65.946 \\
\hline Netherlands [119] & 2006 & regional & East & convenience & 2.200 & 0,2 & $(0.1-0.5) \$$ & & & \\
\hline Poland [120] & 1999 & regional & North & convenience & 2.561 & 1,9 & $(1.4-2.5) \$$ & & 38.135 .876 & 724.582 \\
\hline Romania [121] & $2006-2008$ & nationwide & - & random & 8.039 & 3,5 & $(3.1-3.9) \&$ & & 21.498.616 & 752.452 \\
\hline Spain [122] & 1996 & regional & Catalonia & random & 2.142 & 2,5 & $(1.8-3.2) \&$ & Standardized & 45.828 .172 & 916.563 \\
\hline Spain [123] & 1997-1998 & regional & North & random & 1.170 & 1,6 & $(1.0-2.6) \&$ & & & \\
\hline Sweden [104] & 1991-1994 & regional & Malmö & random & 5.533 & 0,4 & $(0,3-0,6) \$$ & & 9.256 .347 & 37.025 \\
\hline Turkey [105] & 2006-2007 & regional & South West & random & 2.852 & 1,0 & $(0,7-1,4) \$$ & & 71.517 .100 & 1.072 .757 \\
\hline Turkey [106] & $2002-2004$ & regional & Central & convenience & 1.320 & 2,2 & $(1,5-3,1) \$$ & & & \\
\hline Turkey [108] & Not reported & regional & Central & random & 1.095 & 2,1 & $(1,3-3,1) \$$ & & & \\
\hline United Kingdom [124] & 1996 & regional & England\&Wales & residual & 6.401 & 0,7 & $(0,1-0,5) \$$ & & 61634599 & 431442 \\
\hline
\end{tabular}

* No estimate of anti-HCV general population prevalence was found for Austria, Croatia, Cyprus, Denmark, Estonia, Finland, Former Yugoslav Republic of Macedonia, Hungary, Iceland, Ireland, Latvia, Liechtenstein, Lithuania, Luxembourg, Malta, Norway, Portugal, Slovakia, Slovenia and Switzerland.

** Cl Confidence interval.

$\& \mathrm{Cl}$ provided in the original paper.

$\$ \mathrm{Cl}$ estimated by the exact method. 


\section{Cost-effectiveness of HBV and HCV screening}

The search for evidence on cost-effectiveness of HBV and/or HCV screening identified 468 publications. We retrieved the full text for 41 publications (9\%). From the reference lists of included studies 3 additional potentially relevant citations were identified. Of these 44 papers, 13 were considered not relevant following full text review. Two additional publications were excluded $[44,45]$ because they reported on data that were more extensively presented in a third publication [46]. Finally, 29 publications were included in the review of cost-effectiveness of screening (flow chart Additional file 2). No paper studied combined screening for HBV and HCV. Of the 29 papers, 23 used a Markov model (21 with hypothetical data and 2 presented actual screening results). The remaining 6 studies did not use a model and presented costs per case identified or cost per infection prevented. None of the studies included dynamic modeling to take into account effects of reducing transmission by lowering viral load through treatment, behavior change, or HBV vaccination.

\section{General population screening}

One economic analysis of HBsAg screening of the general population (considering in the base case 35 year old males with a $2 \%$ prevalence) found this was cost-effective (incremental cost-effectiveness ratio (ICER) €23.966/quality adjusted life year (QALY)) [47]. Six studies reported on HCV screening and subsequent treatment of the general population [48-53]. Two of these reported costs per LY gained, both concluding $\mathrm{HCV}$ screening of the general population was cost-effective $[48,50]$. Four studies, all from the USA, assessed general population HCV screening by estimating cost per QALY gained [49,51-53]. All studies except one [49] concluded general population HCV screening of adults was cost-effective.

\section{Antenatal screening}

Five economic analyses reported on antenatal HBsAg screening [54-58], presenting estimated costs per LY gained [54-56], costs per case detected and per infant carrier prevented [57] and costs per case detected [58]. The 3 studies presenting costs per LY gained studied the scenario of universal screening of all pregnant women, with vaccination of infants born to HBsAg positive mothers. Studies were published between 1993 and 1997, and none considered antiviral treatment. ICERs ranged from $€ 2,032$ to $€ 26,181$ per LY gained. All studies concluded that universal antenatal HBV screening is cost-effective considering the respective thresholds used. One economic analysis of antenatal HCV screening was found, which considered universal antenatal $\mathrm{HCV}$ screening and treatment of $\mathrm{HCV}$ infection with or without elective cesarean delivery [59]. Neither of these scenarios was considered cost-effective.

\section{PWID}

No economic analysis of HBsAg screening of PWID was found. Ten studies reported on cost-effectiveness of HCV screening and treatment of PWID [43,46,48,60-66]. Seven of these reported estimated costs per QALY. These studies varied widely, including different screening settings, treatments considered, and discount rates. Nevertheless, all 7 studies concluded that HCV screening of PWID was likely to be cost-effective considering the respective thresholds used, with ICERs ranging from $-€ 3.328$ to $€ 41,874$ per QALY.

\section{Migrants}

Four economic analyses of screening migrants for $\mathrm{HBsAg}$ were found [67-70]. One of these compared 4 community outreach screening programs by assessing cost per person screened and cost per HBsAg positive individuals identified, concluding that screening in outpatient settings was the most cost-effective but reached the lowest number of participants [69]. The 3 other studies assessing cost per QALY all concluded migrant screening was cost-effective, with ICERs ranging from $€ 8.694$ [68] to $€ 46.260$ [70]. One economic analysis of HCV screening of migrants was found [66]. In this study, the target group for screening included migrants from countries with a $\mathrm{HCV}$ prevalence $>10 \%$, as well as from other population subgroups. Separate estimates of cost-effectiveness of screening migrants were, however, not presented.

\section{MSM}

We found no economic analysis of HBsAg screening of MSM. One economic analysis of HCV screening of MSM at sexually transmitted disease (STD) clinics concluded that HCV screening of MSM in this setting was not costeffective [43].

\section{STD-clinic attenders}

We did not find any economic analyses of HBsAg screening of STD-clinic attendees, but 2 of HCV screening of STD-clinic attendees $[46,64]$. Universal screening and treatment of UK STD-clinic attendees was assessed as not cost-effective (ICER €125,933/QALY) [46,64]. Among STD-clinic attendees in the US, HCV screening of nonPWID was only cost-effective when restricted to men with $>100$ lifetime sex partners [64].

\section{Prisoners}

No economic analyses of HBsAg screening of prisoners were found. Regarding HCV screening of prisoners, we found two studies both from England and Wales [71,72]. The first study found that asking prisoners about their $\mathrm{HCV}$ and injecting status prior to laboratory testing can considerably reduce the cost per case detected [71]. The second paper found that HCV screening and treatment 
Table 3 Publications included in the cost-effectiveness review $(n=29)$

\begin{tabular}{|c|c|c|c|c|c|c|c|c|c|c|c|}
\hline $\begin{array}{l}\text { First Author, year, } \\
\text { reference }\end{array}$ & Target group & Setting & Country & Infection & Model & Data & Indicator^ $\wedge$ & Result* & $\begin{array}{l}\text { Year of } \\
\text { monetary } \\
\text { value \# }\end{array}$ & Euro in 2010 & Cost-effective? \\
\hline Thomas, 1990 [58] & Pregnant women & Antenatal care & Australia & HBV & None & $\begin{array}{l}\text { Actual } \\
\text { screening }\end{array}$ & $\begin{array}{l}\text { Cost per case } \\
\text { detected }\end{array}$ & $\$ 354(\mathrm{AU})$ & 1988 & $€ 379$ & Yes \\
\hline Audet, 1991 [57] & Pregnant women & Antenatal care & Canada & HBV & None & $\begin{array}{l}\text { Hypothetical } \\
\text { cohort }\end{array}$ & $\begin{array}{l}\text { Cost per case } \\
\text { detected/infant } \\
\text { carrier prevented }\end{array}$ & $\$ 1.693 / \$ 8.915(C A)$ & 1988 & $€ 1.799-€ 9.475$ & Yes, probably \\
\hline Tormans, 1993 [54] & Pregnant women & Antenatal care & Belgium & HBV & Markov & $\begin{array}{l}\text { Hypothetical } \\
\text { cohort }\end{array}$ & Cost per LY gained & BEF 583.581 & 1991 & $€ 22.095$ & Yes \\
\hline Dwyer, 1996 [56] & Pregnant women & Antenatal care & UK & HBV & Markov & $\begin{array}{l}\text { Hypothetical } \\
\text { cohort }\end{array}$ & $\begin{array}{l}\text { Cost per carrier } \\
\text { prevented/LY } \\
\text { gained }\end{array}$ & $£ 2.437 / £ 16.450$ & $\begin{array}{l}\text { Not mentioned } \\
\text { (1996) }\end{array}$ & $€ 3.879-€ 26.181$ & Yes \\
\hline Jordan, 1997 [55] & Pregnant women & Antenatal care & Britain & HBV & Markov & $\begin{array}{l}\text { Hypothetical } \\
\text { cohort }\end{array}$ & $\begin{array}{l}\text { Cost per LY } \\
\text { gained }\end{array}$ & $£ 1.300$ & $\begin{array}{l}\text { Not mentioned } \\
\text { (1997) }\end{array}$ & $€ 2.032$ & Yes \\
\hline Plunkett, 2005 [59] & Pregnant women & Antenatal care & USA & $\mathrm{HCV}$ & Markov & $\begin{array}{l}\text { Hypothetical } \\
\text { cohort }\end{array}$ & Cost per QALY & $\begin{array}{l}\text { No screening } \\
\text { dominant }\end{array}$ & 2003 & n.a. & No \\
\hline Eckman, 2011 [47] & $\begin{array}{l}\text { General population } \\
\text { (35 year old males) }\end{array}$ & Primary care & USA & HBV & Markov & $\begin{array}{l}\text { Hypothetical } \\
\text { cohort }\end{array}$ & Cost per QALY & $\$ 29.232$ (US) & 2008 & $€ 23.966$ & Yes \\
\hline Singer, 2001 [49] & General population & Not specified & USA & $\mathrm{HCV}$ & Markov & $\begin{array}{l}\text { Hypothetical } \\
\text { cohort }\end{array}$ & Cost per QALY & $\begin{array}{l}\text { No screening } \\
\text { dominant }\end{array}$ & 2001 & n.a. & No \\
\hline Nakamura, 2008 [50] & $\begin{array}{l}\text { General population } \\
\text { \& risk groups }\end{array}$ & Not specified & Japan & $\mathrm{HCV}$ & Markov & $\begin{array}{l}\text { Actual } \\
\text { screening }\end{array}$ & $\begin{array}{l}\text { Cost per LY } \\
\text { gained }\end{array}$ & $\$ 848-\$ 4.825$ (US) & 2007 & $€ 726-€ 4.130$ & Yes \\
\hline Loubiere, 2003 [48] & $\begin{array}{l}\text { General population, } \\
\text { IDUs \& other risk } \\
\text { groups }\end{array}$ & Not specified & France & $\mathrm{HCV}$ & Markov & $\begin{array}{l}\text { Hypothetical } \\
\text { cohort }\end{array}$ & $\begin{array}{l}\text { Cost per LY } \\
\text { gained }\end{array}$ & $\begin{array}{l}\$ 4.513 \text { (US) (IDUs)/ } \\
\$ 5.821 \text { (gen pop) }\end{array}$ & 1998 & $€ 4.856 / € 6.263$ & Yes \\
\hline Coffin, 2012 [51] & $\begin{array}{l}\text { General population } \\
\text { (20-69 y.o. and } \\
1945-1965)\end{array}$ & Not specified & USA & $\mathrm{HCV}$ & Markov & $\begin{array}{l}\text { Hypothetical } \\
\text { cohort }\end{array}$ & Cost per QALY & $\$ 7.900-\$ 5.400$ (US) & 2010 & $€ 6.376-€ 4.358$ & Yes \\
\hline McGarry, 2012 [52] & $\begin{array}{l}\text { General population } \\
\text { (born 1946-1970) }\end{array}$ & Not specified & USA & $\mathrm{HCV}$ & Markov & $\begin{array}{l}\text { Hypothetical } \\
\text { cohort }\end{array}$ & Cost per QALY & $\$ 37.720$ (US) & 2010 & $€ 30.444$ & Yes \\
\hline Rein, 2012 [53] & $\begin{array}{l}\text { General population } \\
\text { (born 1945-1965) }\end{array}$ & Not specified & USA & $\mathrm{HCV}$ & Markov & $\begin{array}{l}\text { Hypothetical } \\
\text { cohort }\end{array}$ & Cost per QALY & $\$ 15.700$ (US) & 2009 & $€ 12.976$ & Yes \\
\hline Ruggeri, 2011 [73] & High risk groups & Not specified & Italy & HBV & Markov & $\begin{array}{l}\text { Hypothetical } \\
\text { cohort }\end{array}$ & Cost per QALY & $€ 18.255$ (IT) & 2004 & $€ 52.885$ & Yes \\
\hline Hutton, 2007 [67] & Migrants & Not specified & USA & HBV & Markov & $\begin{array}{l}\text { Hypothetical } \\
\text { cohort }\end{array}$ & Cost per QALY & $\$ 36.088$ (US) & 2006 & $€ 31.692$ & Yes \\
\hline Veldhuijzen, 2010 [68] & Migrants & $\begin{array}{l}\text { Population } \\
\text { based }\end{array}$ & Netherlands & HBV & Markov & $\begin{array}{l}\text { Hypothetical } \\
\text { cohort }\end{array}$ & Cost per QALY & $€ 8.966$ (NI) & 2009 & $€ 8.694$ & Yes \\
\hline Rein, 2011 [69] & Migrants & $\begin{array}{l}5 \text { settings } \\
\text { compared }\end{array}$ & USA & HBV & None & $\begin{array}{l}\text { Actual } \\
\text { screening }\end{array}$ & $\begin{array}{l}\text { Cost per case } \\
\text { detected }\end{array}$ & \$609-\$4.657 (US) & 2008 & $€ 499-€ 3.818$ & Yes \\
\hline
\end{tabular}


Table 3 Publications included in the cost-effectiveness review $(\mathbf{n}=29)$ (Continued)

\begin{tabular}{|c|c|c|c|c|c|c|c|c|c|c|c|}
\hline Wong, 2011 [70] & Migrants & Not specified & Canada & HBV & Markov & $\begin{array}{l}\text { Hypothetical } \\
\text { cohort }\end{array}$ & Cost per QALY & $\$ 69.209$ (CA) & 2008 & $€ 46.260$ & Yes, moderately \\
\hline Leal, 1999 [60] & IDUs & Drug services & UK & $\mathrm{HCV}$ & Markov & $\begin{array}{l}\text { Hypothetical } \\
\text { cohort }\end{array}$ & Cost per QALY & $£ 9.300$ & 1997 & $€ 14.540$ & Yes \\
\hline Castelnuovo, 2006 [61] & IDUs & Various & UK & $\mathrm{HCV}$ & Markov & $\begin{array}{l}\text { Hypothetical } \\
\text { cohort }\end{array}$ & Cost per QALY & $£ 15.493-£ 20.083$ & 2004 & $€ 22.172 € € 28.741$ & Yes \\
\hline $\begin{array}{l}\text { Thompson Coon, } \\
2006 \text { [62] }\end{array}$ & IDUs & Primary care & UK & $\mathrm{HCV}$ & Markov & $\begin{array}{l}\text { Hypothetical } \\
\text { cohort }\end{array}$ & Cost per QALY & $£ 16.493$ & $2002 / 2003$ & $€ 24.245$ & Yes \\
\hline Kerr, 2009 [43] & IDUs \& MSM & STD clinic & Scotland & $\mathrm{HCV}$ & None & $\begin{array}{l}\text { Actual } \\
\text { screening }\end{array}$ & $\begin{array}{l}\text { Cost per case } \\
\text { detected }\end{array}$ & $\begin{array}{l}£ 170 \text { (IDU)/ } \\
£ 15.000 \text { (MSM) }\end{array}$ & $\begin{array}{l}\text { Not mentioned } \\
\text { (2009) }\end{array}$ & $€ 215 € € 18.975$ & $\begin{array}{l}\text { Yes (IDU), No } \\
\text { (MSM) }\end{array}$ \\
\hline Josset, 2004 [63] & $\begin{array}{l}\text { IDUs \& other risk } \\
\text { groups }\end{array}$ & Primary care & France & $\mathrm{HCV}$ & None & $\begin{array}{l}\text { Actual } \\
\text { screening }\end{array}$ & $\begin{array}{l}\text { Cost per case } \\
\text { detected }\end{array}$ & not reported & Not mentioned & n.a. & Not stated \\
\hline Stein, 2004 [44] & $\begin{array}{l}\text { IDUs \& other risk } \\
\text { groups }\end{array}$ & $\begin{array}{l}\text { STD clinic/ } \\
\text { drug services }\end{array}$ & UK & $\mathrm{HCV}$ & Markov & $\begin{array}{l}\text { Hypothetical } \\
\text { cohort }\end{array}$ & Cost per QALY & $\begin{array}{l}£ 28.120 \text { (IDUs)/ } \\
£ 84.570 \text { (GUM) }\end{array}$ & 2001 & $\begin{array}{l}€ 41.874- \\
€ 125.933\end{array}$ & $\begin{array}{l}\text { Yes (IDUs)/No } \\
\text { (GUM) }\end{array}$ \\
\hline Honeycutt, 2007 [64] & $\begin{array}{l}\text { IDUs \& other risk } \\
\text { groups }\end{array}$ & STD clinic & USA & $\mathrm{HCV}$ & None & $\begin{array}{l}\text { Hypothetical } \\
\text { cohort }\end{array}$ & $\begin{array}{l}\text { Cost per case } \\
\text { detected }\end{array}$ & \$54 (US) & 2006 & $€ 47$ & Yes \\
\hline Tramarin, 2008 [65] & $\begin{array}{l}\text { IDUs \& other risk } \\
\text { groups }\end{array}$ & Not specified & Italy & $\mathrm{HCV}$ & Markov & $\begin{array}{l}\text { Hypothetical } \\
\text { cohort }\end{array}$ & Cost per QALY & $€ 3.132(\mathrm{IT})$ & 2007 & $€ 3.328$ & Yes \\
\hline Helsper, 2012 [66] & $\begin{array}{l}\text { IDUs \& other risk } \\
\text { groups incl } \\
\text { migrants }\end{array}$ & $\begin{array}{l}\text { Primary care/ } \\
\text { drug services }\end{array}$ & Netherlands & $\mathrm{HCV}$ & Markov & $\begin{array}{l}\text { Actual } \\
\text { screening }\end{array}$ & Cost per QALY & $€ 7.321(\mathrm{NL})$ & 2007 & $€ 7.327$ & Yes \\
\hline Sutton, 2006 [71] & Prisoners & Prison & UK & $\mathrm{HCV}$ & Markov & $\begin{array}{l}\text { Hypothetical } \\
\text { cohort }\end{array}$ & $\begin{array}{l}\text { Cost per case } \\
\text { detected }\end{array}$ & $£ 2,102-£ 3,107$ & 2004 & $€ 3.008-€ 4.446$ & Yes \\
\hline Sutton, 2008 [72] & Prisoners & Prison & UK & $\mathrm{HCV}$ & Markov & $\begin{array}{l}\text { Hypothetical } \\
\text { cohort }\end{array}$ & Cost per QALY & $£ 54.852$ & 2004 & $€ 78.498$ & No \\
\hline
\end{tabular}

* Results of most favorable scenario reported here.

\# Where the paper did not quote year of monetary value the year of publication was used to convert the results into 2010 Euros [34]

t available.

$\wedge$ QALY, Quality adjusted life year; $L Y$, Life year. 
of prisoners was not cost-effective (cost per QALY $€ 78,498)$ [72].

\section{Other high-risk groups}

One study assessed HBsAg screening of high-risk groups currently recommended for screening in Italy as cost-effective [73]. However, no costs for the screening programme were taken into account and compliance was set at an unrealistic $100 \%$. Five economic analyses of HCV screening of other population subgroups were found $[48,50,63,65,66,74]$. Both studies that considered screening programmes targeting several population subgroups concluded that the specific programmes considered were potentially cost-effective $[50,66]$. Josset et al. reported estimated costs per positive test result for 6 screening scenarios, which varied regarding population subgroups targeted [63]. Analyses of HCV screening of people transfused before 1991 in France and of people with a history of surgery in Italy both concluded this was not cost-effective $[48,65]$.

\section{Discussion and conclusions Prevalence of hepatitis B and C}

The general population prevalence of chronic HBV and $\mathrm{HCV}$ infection varies widely between European countries, with those in the south and east of the European Union and in Turkey having a much higher prevalence than those in northwestern Europe. Among countries for which data were available for both infections, Romania stands out, with high prevalence for both HBV and HCV. In contrast, Belgium, Sweden, Germany, and The Netherlands have low-population prevalence for both infections. Results from a study published after our literature search was completed suggest that France also belongs to this latter category [24]. A recent systematic review of chronic HBV prevalence in Turkey was consistent with our findings reporting a similar west to east gradient [75]. For HCV, Italy had the highest estimated population prevalence, much higher than its estimated HBV prevalence. Epidemiologic and phylogenetic assessments suggest that this may have been caused by a period of frequent iatrogenic transmission that took place around the 1950s [76]. Without screening and early treatment, these infections will lead to a considerable disease burden and many deaths due to liver disease in the coming decades. Given that HBV and HCV disproportionately affect disadvantaged groups and less affluent countries in Europe, these infections will also contribute to increasing inequalities in health.

For the majority of countries, data on the general population prevalence of $\mathrm{HBV}$ or $\mathrm{HCV}$ were lacking. Availability of sufficiently recent estimates is necessary to be able to prioritize primary and secondary prevention of HBV and $\mathrm{HCV}$ among other public health interventions, to evaluate control measures, and for health care planning. Estimates of prevalence obtained from blood-donor and antenatal screening were found to differ substantially from general population estimates. Within countries, the prevalence of HBsAg and anti-HCV-Ab among PWID, MSM, and migrants was much higher than the corresponding prevalence in the general population, with only a few exceptions. The higher HBsAg prevalence among migrants was confirmed by a recent systematic review [77]. Of the high-risk groups considered, PWID had by far the highest prevalence, particularly for HCV.

\section{Cost-effectiveness of screening for hepatitis $B$ and $C$}

The search for evidence on cost-effectiveness of screening was consistent with the prevalence review: For all three population subgroups with evidence of increased $\mathrm{HBV}$ and $\mathrm{HCV}$ prevalence compared to the general population (PWID, MSM and migrants) economic analyses of screening were found. This resulted in evidence that HCV screening of PWID and HBsAg screening of pregnant women and migrants are cost-effective interventions to reduce the burden of disease due to viral hepatitis. HCV screening of pregnant women and comprehensive screening of all STD-clinic attendees is probably not cost-effective, although there may be exceptions for some specific local or subpopulation conditions. General population screening for HCV was found cost-effective in the US 'baby-boom generation' (born 1945-1965). For other programs, including HBV screening of PWID, HCV screening migrants, $\mathrm{HBV}$ and $\mathrm{HCV}$ screening of prisoners and MSM, the evidence found in this systematic review was insufficient to draw conclusions.

\section{Screening of PWID}

The strongest evidence regarding cost-effectiveness was available for HCV screening of PWID. The wide range in ICER estimates may be partly explained by differing definitions of PWID, whereby some studies may include former PWID. It is unclear to what extent PWID in Europe are offered HCV screening and are successfully referred once found to be positive. HCV screening programmes for PWID exist in only 16 of the 29 European Union/European Economic Area countries reviewed in 2009 [26] whereby testing coverage and referral to treatment often remain poor [78]. On the other hand, several countries without screening programmes report adequate HCV testing of PWID [79]. This apparent discrepancy may be explained by a lack of definition of what a screening programme entails and/or the possibility that the 2009 review has missed screening programmes of PWID. Nevertheless, optimizing implementation of testing guidelines for PWID and monitoring of this group are among the highest priorities $[80,81]$. 


\section{Antenatal screening}

Regarding HBsAg screening of pregnant women it is likely that it would be even more economically favourable if antiviral treatment of the mother was considered. European countries that currently have selective or no antenatal HBsAg screening programmes, including Bulgaria, Lithuania, Luxembourg, Romania, and Norway, should consider implementing universal antenatal screening [26]. This holds even if these countries have universal infant HBV-vaccination programme with an at-birth dose of vaccine, since prevention of perinatal HBV transmission requires the first dose of vaccine to be given within 24 hours and a very high uptake of vaccination. In addition, providing hepatitis B immunoglobulin is of additional effectiveness $[82,83]$.

\section{Migrant screening}

The four publications examining HBsAg screening of migrants born in endemic countries (HBsAg prevalence $\geq 2 \%$ ) suggest this is cost-effective. Main determinants of ICER were the proportion of eligible people starting treatment, disease progression rates with and without treatment, and costs of treatment $[67,68]$. Further research should focus on these areas of uncertainty, as well as on how to optimize participation in screening and referral pathways [69,84]. Given that HCV could be tested using the same blood sample and that migrants generally have higher $\mathrm{HCV}$ prevalence than the indigenous population in European countries, an economic assessment of combined $\mathrm{HBV} / \mathrm{HCV}$ screening for migrants is a priority $[85,86]$.

\section{General population screening}

The only study found that considered general population screening for HBsAg [47], suggested this would be cost-effective in populations with a prevalence above $0.3 \%$. This includes nearly all European countries. However, the study considered only men, included no costs for the screening programme (except for a blood test and consultation) and made unrealistic assumptions regarding compliance with treatment. The evidence for general population HBsAg screening can therefore be considered weak. Regarding general population screening for anti-HCV recent studies mainly from the USA suggest this is cost-effective, particularly when targeted at high-prevalence birth cohorts, the so-called baby-boomers (1945-1965). In response to this, CDC has recommended these cohorts to be offered screening [87]. In Europe, a French study from 2003 suggested HCV screening for the general population could be cost-effective [48]. More evidence on general population HCV screening needed is needed for European countries, especially for those with a relatively high prevalence.

\section{Limitations}

The main limitation of our review is regarding the comparability of the estimates found. First this is limited since different laboratory tests were used, particularly for $\mathrm{HCV}$ where antibody assay validity has improved in recent years. Second, prevalence estimates were not always standardized by age and sex. Lastly, the definition and sampling of the high risk population groups are likely to influence prevalence estimates found both for these groups as for the general population. A limitation of the cost-effectiveness studies is that most analyses used Markov modeling, necessary since the disease outcomes of chronic HBV and $\mathrm{HCV}$ infection take several decades to develop. When not accounting for co-morbidities such as excess of alcohol intake, these models can overestimate the effects of screening and treatment by having too optimistic assumptions about life expectancy. On the other hand, effects of screening are underestimated since these models do not allow quantifying the effect of reduced transmission by lowering viral load due to antiviral treatment and potential behavior change. Since these effects can be considerable [13-15], dynamic models assessing the effects of screening and treatment need to be developed. This is likely to be of particular relevance for population subgroups where not only the prevalence, but also the incidence, is increased compared to the general population, such as MSM (HBV) and PWID (HCV). A recent study from Martin et al. did include indirect effects of treating HCV infections in PWID. It was not included in our review since it assessed cost-effectiveness of treatment rather than of screening and treatment [88].

Lastly, methods, assumptions, and quality varied between studies, making it difficult to compare results and limiting the possibilities of carrying out meta-analyses. Guidelines such as those developed for economic analyses of vaccination programmes may be helpful to improve the quality of studies $[89,90]$.

\section{Conclusions}

Available data suggest a wide variation in prevalence of chronic HBV and HCV infection between countries in Europe. Countries in the south and east of the European Union and in Turkey have a much higher prevalence for chronic HBV and HCV than countries in northwestern Europe. For the majority of countries data on the general population prevalence of $\mathrm{HBV}$ or $\mathrm{HCV}$ are lacking. Within countries, the prevalence of $\mathrm{HBsAg}$ and anti-HCV-Ab among PWID, MSM, and migrants is generally much higher than the general population prevalence. Considerable health benefits can be gained cost-effectively by antiHCV-Ab screening of PWID. HBsAg screening of pregnant women and migrants is also very likely cost-effective. Appraisals of the evidence for screening the general population in mid- and highly endemic countries in Europe and 
of combined HBV/HCV screening are needed. Future costeffectiveness analyses may need to take the effect of antiviral treatment on preventing $\mathrm{HBV}$ and $\mathrm{HCV}$ transmission into account.

\section{Ethics statement}

An ethics statement was not required for this work.

\section{Endnotes}

All 27 EU member states, 4 EEA/EFTA countries (Norway, Iceland, Liechtenstein and Switzerland) and 3 EU enlargement countries (Croatia, the former Yugoslav Republic of Macedonia and Turkey).

\section{Additional files}

Additional file 1: Search strategy. S1. 1 Prevalence studies, General population, 34 countries European region, Prevalence in 5 specific population subgroups, 34 countries European Region, S1. 2 Costeffectiveness studies.

Additional file 2: PRISMA flow diagrams [114]. S2.1 Systematic review of seroprevalence of HBsAg and anti-HCV-Ab, S2. 2 Systematic review of cost-effectiveness of screening for chronic HBV and HCV infection.

Additional file 3: $\mathrm{HBsAg}$ and anti-HCV-Ab prevalence estimates combined and in population subgroups, by country, European neighbourhood. Figure S2. Summary of HBsAg and anti-HCV-Ab prevalence profiles in Europe, 2000-2009. Figure S3. 1a First-time blood donors: HBsAg prevalence (\%) by country, Europe, 2000-2009. Figure S3. 1b First-time blood donors: anti-HCV-Ab prevalence (\%) by country, Europe, 2000-2009. Figure S3. 2a Pregnant women: HBsAg prevalence (\%) by country, Europe, 2000-2009. Figure S3. 2b Pregnant women: anti-HCV-Ab prevalence (\%) by country, Europe, 2000-2009. Figure S3. 3a People who inject drugs (PWID): HBsAg prevalence (\%) by country, Europe, 2000-2009. Figure S3. 3b PWID: anti-HCV-Ab prevalence (\%)by country, Europe, 2000-2009.

Additional file 4: Table S3. 1a First-time blood donors: HBsAg prevalence (\%) by country, Europe, 2000-2009. Table S3. 1b First-time blood donors: anti-HCV-Ab prevalence (\%) by country, Europe, 20002009. Table S3. 2a Pregnant women: HBsAg prevalence (\%) by country, Europe, 2000-2009. Table S3. 2b Pregnant women: anti-HCV-Ab prevalence (\%) by country, Europe, 2000-2009. Table S3. 3a PWID: HBsAg prevalence (\%) by country, Europe, 2000-2009, Table S3. 3b PWID: anti-HCV-Ab prevalence (\%) by country, Europe, 2000-2009. Table S3. 4a Migrants: HBsAg prevalence (\%) by country of residence, Europe, 2000-2009 Table S3. 4b Migrants: anti-HCV-Ab prevalence (\%) by country of residence, Europe, 2000-2009.

\section{Competing interests}

The authors declare that they have no competing interests.

\section{Authors' contributions}

$\mathrm{SH}, \mathrm{IV}, \mathrm{ML}$ and MS designed the study. SH and IV carried out the systematic review and wrote the manuscript. LW provided and interpreted the data on PWID. TL assisted with the economic analyses. All authors contributed to interpreting the data and writing the manuscript. All authors read and approved the final manuscript.

\section{Acknowledgements}

The authors would like to thank J. Alblas, J. Ewijk, W. ten Have, and A. van Ginkel for their contributions to this study, H. Giesbers for help with the maps, and S. Landry for editing the final manuscript.

\section{Financial disclosure}

This study was commissioned by the European Centre for Disease Control and Prevention (ECDC), Stockholm, Sweden (Contract no: ECDC/09/1711) and the National Institute for Public Health and The Environment (RIVM), The Netherlands. ECDC and RIVM staff contributed to the design, implementation, analyses and reporting of this study.

\section{Funding}

This study was commissioned by the European Centre for Disease Control and Prevention, Sweden.

\section{Author details}

${ }^{1}$ Centre for Infectious Disease Control, National Institute for Public Health and the Environment (RIVM), PO Box 1, 3720 BA Bilthoven, The Netherlands. ${ }^{2}$ Municipal Public Health Service Rotterdam-Rijnmond, PO Box 70032, 3000 LP Rotterdam, The Netherlands. ${ }^{3}$ European Centre for Disease Prevention and Control (ECDC), Tomtebodavägen 11a, 17183 Stockholm, Sweden. ${ }^{4}$ European Monitoring Centre for Drugs and Drug Addiction (EMCDDA), Cais do Sodré, 1249-289 Lisbon, Portugal.

Received: 24 September 2012 Accepted: 21 March 2013 Published: 18 April 2013

\section{References}

1. Lok AS: Chronic hepatitis B. N Engl J Med 2002, 346:1682-1683.

2. Seeff LB: Natural history of chronic hepatitis C. Hepatology 2002, 36:S35-S46.

3. Medley GF, Lindop NA, Edmunds WJ, Nokes DJ: Hepatitis-B virus endemicity: heterogeneity, catastrophic dynamics and control. Nat Med 2001, 7:619-624.

4. Dienstag JL: Hepatitis B virus infection. N Engl J Med 2008, 359:1486-1500.

5. World Health Organization: Hepatitis B. 2008. http:/www.who.int/mediacentre/ factsheets/fs204/en/.

6. World Health Organization: Hepatitis C. 2011. http://ecdc.europa.eu/en/ publications/Publications/TER_100914_Hep_B_C\%20_EU_neighbourhood.pdf.

7. Plotkin O: Vaccine. Amsterdam: Saunders Elsevier; 2008

8. Kanwal F, Gralnek IM, Martin P, Dulai GS, Farid M, Spiegel BM: Treatment alternatives for chronic hepatitis $B$ virus infection: a cost-effectiveness analysis. Ann Intern Med 2005, 142:821-831.

9. Takeda A, Jones J, Shepherd J, Davidson P, Price A: A systematic review and economic evaluation of adefovir dipivoxil and pegylated interferonalpha-2a for the treatment of chronic hepatitis B. J Viral Hepat 2007, 14:75-88.

10. Shepherd J, Brodin HF, Cave CB, Waugh NR, Price A, Gabbay J: Clinical- and cost-effectiveness of pegylated interferon alfa in the treatment of chronic hepatitis C: a systematic review and economic evaluation. Int J Technol Assess Health Care 2005, 21:47-54.

11. European Association For The Study Of The Liver: EASL Clinical Practice Guidelines: Management of chronic hepatitis B. J Hepatol 2009, 50:227-242.

12. European Association For The Study Of The Liver: EASL Clinical Practice Guidelines: management of hepatitis C virus infection. J Hepatol 2011, 55:245-264.

13. Martin NK, Vickerman P, Hickman M: Mathematical modelling of hepatitis C treatment for injecting drug users. J Theor Biol 2011, 274:58-66.

14. Martin NK, Vickerman P, Foster GR, Hutchinson SJ, Goldberg DJ, Hickman M: Can antiviral therapy for hepatitis $\mathrm{C}$ reduce the prevalence of $\mathrm{HCV}$ among injecting drug user populations? A modeling analysis of its prevention utility. J Hepatol 2011, 54:1137-1144.

15. Cohen MS, Chen YQ, McCauley M, Gamble T, Hosseinipour MC, Kumarasamy N, Hakim JG, Kumwenda J, Grinsztejn B, Pilotto JH, et al: Prevention of HIV-1 infection with early antiretroviral therapy. N Engl J Med 2011, 365:493-505.

16. Fattovich G, Stroffolini T, Zagni I, Donato F: Hepatocellular carcinoma in cirrhosis: incidence and risk factors. Gastroenterology 2004, 127:S35-S50.

17. Missiha SB, Ostrowski M, Heathcote EJ: Disease progression in chronic hepatitis C: modifiable and nonmodifiable factors. Gastroenterology 2008 134:1699-1714.

18. Wasley A, Finelli L, Bell B, Alter M: The knowledge and behaviors of HCVinfected persons identified in a seroprevalence survey, USA, 2001-2002 [abstract]. J Clin Virol 2006, 36:S198-S199. 
19. Richter C, Beest GT, Sancak I, Aydinly R, Bulbul K, Laetemia-Tomata F, DE Leeuw M, Waegemaekers T, Swanink C, Roovers E: Hepatitis B prevalence in the Turkish population of Arnhem: implications for national screening policy? Epidemiol Infect 2012, 140:724-730.

20. Kwiatkowski CF, Fortuin CK, Booth RE: The association between knowledge of hepatitis $C$ virus status and risk behaviors in injection drug users. Addiction 2002, 97:1289-1294.

21. Culver DH, Alter MJ, Mullan RJ, Margolis HS: Evaluation of the effectiveness of targeted lookback for HCV infection in the United States-interim results. Transfusion 2000, 40:1176-1181.

22. Hagan H, Campbell J, Thiede H, Strathdee S, Ouellet L, Kapadia F, Hudson S, Garfein RS: Self-reported hepatitis $C$ virus antibody status and risk behavior in young injectors. Public Health Rep 2006, 121:710-719.

23. Hutchinson SJ, Roy KM, Wadd S, Bird SM, Taylor A, Anderson E, Shaw L, Codere G, Goldberg DJ: Hepatitis C virus infection in Scotland: epidemiological review and public health challenges. Scott Med J 2006, 51:8-15.

24. Meffre $C$, Le Strat $Y$, Delarocque-Astagneau $E$, Dubois F, Antona D, Lemasson JM, Warszawski J, Steinmetz J, Coste D, Meyer JF, et al: Prevalence of hepatitis $B$ and hepatitis $C$ virus infections in France in 2004: social factors are important predictors after adjusting for known risk factors. J Med Virol 2010, 82:546-555.

25. Hahné SJ, de Melker HE, Kretzschmar M, Mollema L, vd Klis FR, vd Sande MA, Boot HJ: Prevalence of hepatitis B virus infection in The Netherlands in 1996 and 2007. Epidemiol Infect 2012, 140:1469-1480.

26. European Centre for Disease Prevention and Control: Surveillance and prevention of hepatitis B and C in Europe. Stockholm; 2010. http://www.edqm.eu/medias/ fichiers/The_Collection_Testing_and_Use_of_Blood_and_Blood_.pdf.

27. Custer B, Sullivan SD, Hazlet TK, Iloeje U, Veenstra DL, Kowdley KV: Global epidemiology of hepatitis B virus. J Clin Gastroenterol 2004, 38:S158-S168.

28. Muhlberger N, Schwarzer R, Lettmeier B, Sroczynski G, Zeuzem S, Siebert U: HCV-related burden of disease in Europe: a systematic assessment of incidence, prevalence, morbidity, and mortality. BMC Pub/ Health 2009, 9:34.

29. Esteban JI, Sauleda S, Quer J: The changing epidemiology of hepatitis C virus infection in Europe. J Hepatol 2008, 48:148-162.

30. Sroczynski G, Esteban E, Conrads-Frank A, Schwarzer R, Muhlberger N, Wright $D$, Zeuzem S, Siebert U: Long-term effectiveness and cost-effectiveness of screening for hepatitis C virus infection. Eur J Publ Health 2009, 19:245-253.

31. van der Poel C, Janssen MP, Borkent-Raven B: The collection, testing and use of blood and blood products in Europe in. 2005. http://www.edqm.eu/ medias/fichiers/The_Collection_Testing_and_Use_of_Blood_and_Blood_pdf.

32. European Monitoring Centre for Drugs and Drugs Addiction (EMCDDA): Drug related infectious diseases, Statistical Bulletin. 2010. http://www.emcdda. europa.eu/stats10.

33. Nelson PK, Mathers BM, Cowie B, Hagan H, Des Jarlais D, Horyniak D, Degenhardt L: Global epidemiology of hepatitis B and hepatitis $C$ in people who inject drugs: results of systematic reviews. Lancet 2011 378:571-583.

34. OECD: Purchasing Power Parities (PPPS). 2012. Available from http://epp.eurostat. ec.europa.eu/tgm/table.do?tab=table\&language $=e n \& p c o d e=t e c 00118$ \&tableSelection=1\&footnotes=yes\&labeling=labels\&plugin=1. Accessed 13 April 2012.

35. Eurostat: Inflation rates. 2012. Available from http://appsso.eurostat.ec. europa.eu/nui/show.do. Accessed 2 March 2012

36. Eurostat: Population size by country. 2009. Available from http://epp. eurostat.ec.europa.eu/portal/page/portal/statistics/search_database. Accessed 22 July 2009.

37. Liberati A, Altman DG, Tetzlaff J, Mulrow C, Gotzsche PC, loannidis JP, Clarke M, Devereaux PJ, Kleijnen J, Moher D: The PRISMA statement for reporting systematic reviews and meta-analyses of studies that evaluate health care interventions: explanation and elaboration. PLoS Med 2009, 6:e1000100.

38. Roy KM, Goldberg DJ, Wilson K, Cameron SO: Vaccination induced immunity to the hepatitis B virus among high-risk groups in Glasgow 1993-2001: evaluating the effectiveness of the United Kingdom's selective immunisation policy. Scott Med J 2008, 53:13-17.

39. McMillan A: The changing prevalence of hepatitis $B$ virus infection among men who have sex with men who attended a sexually transmitted infections clinic in Edinburgh, Scotland between 1989 and 2003. Int J STD AIDS 2006, 17:539-542

40. Christiansen MA, Lowhagen GB: Sexually transmitted diseases and sexual behavior in men attending an outpatients' clinic for gay men in Gothenburg, Sweden. Acta Derm Venereol 2000, 80:136-139.
41. Baaten GG, Sonder GJ, Dukers NH, Coutinho RA, van den Hoek JA: Population-based study on the seroprevalence of hepatitis $A, B$, and C virus infection in Amsterdam, 2004. J Med Virol 2007, 79:1802-1810.

42. Cavlek TV, Margan IG, Lepej SZ, Kolaric B, Vince A: Seroprevalence, risk factors, and hepatitis $C$ virus genotypes in groups with high-risk sexual behavior in Croatia. J Med Virol 2009, 81:1348-1353.

43. Kerr RS, Fernando I, Templeton K, Flynn B: Hepatitis C screening in genitourinary clinic attendees. Int J STD AIDS 2009, 20:808.

44. Stein K, Dalziel K, Walker A, Jenkins B, Round A, Royle P: Screening for Hepatitis $C$ in injecting drug users: a cost utility analysis. J Public Health (Oxf) 2004, 26:61-71.

45. Stein K, Dalziel K, Walker A, Jenkins B, Round A, Royle P: Screening for hepatitis $C$ in genito-urinary medicine clinics: a cost utility analysis. J Hepatol 2003, 39:814-825.

46. Stein K, Dalziel K, Walker A, McIntyre L, Jenkins B, Horne J, Royle P, Round A: Screening for hepatitis $C$ among injecting drug users and in genitourinary medicine clinics: systematic reviews of effectiveness, modelling study and national survey of current practice. Health Technol Assess 2002, 6:1-122

47. Eckman MH, Kaiser TE, Sherman KE: The cost-effectiveness of screening for chronic hepatitis B infection in the United States. Clin Infect Dis 2011, 52:1294-1306.

48. Loubiere S, Rotily M, Moatti JP: Prevention could be less cost-effective than cure: the case of hepatitis $C$ screening policies in France. Int J Technol Assess Health Care 2003, 19:632-645.

49. Singer ME, Younossi ZM: Cost effectiveness of screening for hepatitis $C$ virus in asymptomatic, average-risk adults. Am J Med 2001, 111:614-621.

50. Nakamura J, Terajima K, Aoyagi Y, Akazawa K: Cost-effectiveness of the national screening program for hepatitis $C$ virus in the general population and the high-risk groups. Tohoku J Exp Med 2008, 215:33-42.

51. Coffin PO, Scott JD, Golden MR, Sullivan SD: Cost-effectiveness and population outcomes of general population screening for hepatitis $C$. Clin Infect Dis 2012, 54:1259-1271.

52. McGarry $\amalg$, Pawar VS, Panchmatia HR, Rubin JL, Davis GL, Younossi ZM, Capretta JC, O'Grady MJ, Weinstein MC: Economic model of a birth cohort screening program for hepatitis C virus. Hepatology 2012, 55:1344-1355

53. Rein DB, Smith BD, Wittenborn JS, Lesesne SB, Wagner LD, Roblin DW, Patel N, Ward JW, Weinbaum CM: The cost-effectiveness of birth-cohort screening for hepatitis C antibody in U.S. primary care settings. Ann Intern Med 2012, 156:263-270.

54. Tormans G, Van Damme P, Carrin G, Clara R, Eylenbosch W: Cost-effectiveness analysis of prenatal screening and vaccination of hepatitis $B$ virus - the case of Belgium. Soc Sci Med 1993, 37:173-181.

55. Jordan R, Law M: An appraisal of the efficacy and cost-effectiveness of antenatal screening for hepatitis B. J Med Screen 1997, 4:117-127.

56. Dwyer MJ, McIntyre PG: Ante-natal screening for hepatitis B surface antigen: an appraisal of its value in a low prevalence area. Epidemiol Infect 1996, 117:121-131.

57. Audet AM, Delage G, Remis RS: Screening for HBsAg in pregnant women: a cost analysis of the universal screening policy in the province of Quebec. Can J Publ Health 1991, 82:191-195.

58. Thomas IL: Cost effectiveness of antenatal hepatitis B screening and vaccination of infants. Aust N Z J Obstet Gynaecol 1990, 30:331-335.

59. Plunkett $B A$, Grobman WA: Routine hepatitis $C$ virus screening in pregnancy: a cost-effectiveness analysis. Am J Obstet Gynecol 2005, 192:1153-1161.

60. Leal $P$, Stein $K$, Rosenberg W: What is the cost utility of screening for hepatitis $C$ virus (HCV) in intravenous drug users? J Med Screen 1999, 6:124-131.

61. Castelnuovo E, Thompson-Coon J, Pitt M, Cramp M, Siebert U, Price A, Stein K: The cost-effectiveness of testing for hepatitis $C$ in former injecting drug users. Health Technol Assess 2006, 10:iii-xii. 1.

62. Thompson CJ, Castelnuovo E, Pitt M, Cramp M, Siebert U, Stein K: Case finding for hepatitis $C$ in primary care: a cost utility analysis. Fam Pract 2006, 23:393-406.

63. Josset V, Torre JP, Tavolacci MP, Rossem-Magnani V, Anselme K, Merle V, Godart J, Libert A, Ladner J, Czernichow P: Efficiency of hepatitis C virus screening strategies in general practice.[see comment]. Gastroenterol Clin Biol 2004, 28:351-357.

64. Honeycutt AA, Harris JL, Khavjou O, Buffington J, Jones TS, Rein DB: The costs and impacts of testing for hepatitis $C$ virus antibody in public STD clinics. Publ Health Rep 2007, 122(Suppl 2):55-62.

65. Tramarin A, Gennaro N, Compostella FA, Gallo C, Wendelaar Bonga LJ, Postma MJ: HCV screening to enable early treatment of hepatitis C: a 
mathematical model to analyse costs and outcomes in two populations. Curr Pharm Des 2008, 14:1655-1660.

66. Helsper CW, Borkent-Raven BA, DE Wit NJ, VAN Essen GA, Bonten MJ, Hoepelman Al, Janssen MP, De Wit GA: Cost-effectiveness of targeted screening for hepatitis C in The Netherlands. Epidemiol Infect 2012, 140:58-69.

67. Hutton DW, Tan D, So SK, Brandeau ML: Cost-effectiveness of screening and vaccinating Asian and Pacific Islander adults for hepatitis B. Ann Intern Med 2007, 147:460-469.

68. Veldhuijzen IK, Toy M, Hahne SJ, De Wit GA, Schalm SW, de Man RA, Richardus JH: Screening and early treatment of migrants for chronic hepatitis B virus infection is cost-effective. Gastroenterology 2010, 138:522-530.

69. Rein DB, Lesesne SB, Smith BD, Weinbaum CM: Models of communitybased hepatitis $B$ surface antigen screening programs in the U.S. and their estimated outcomes and costs. Publ Health Rep 2011, 126:560-567.

70. Wong WW, Woo G, Jenny HE, Krahn M: Cost effectiveness of screening immigrants for hepatitis B. Liver Int 2011, 31:1179-1190.

71. Sutton AJ, Edmunds WJ, Gill ON: Estimating the cost-effectiveness of detecting cases of chronic hepatitis $C$ infection on reception into prison. BMC Publ Health 2006, 6:170.

72. Sutton AJ, Edmunds WJ, Sweeting MJ, Gill ON: The cost-effectiveness of screening and treatment for hepatitis C in prisons in England and Wales: a cost-utility analysis. J Viral Hepat 2008, 15:797-808.

73. Ruggeri M, Cicchetti A, Gasbarrini A: The cost-effectiveness of alternative strategies against HBV in Italy. Health Policy 2011, 102:72-80.

74. Fabris P, Baldo V, Baldovin T, Bellotto E, Rassu M, Trivello R, Tramarin A, Tositti G, Floreani A: Changing epidemiology of HCV and HBV infections in Northern Italy: a survey in the general population. J Clin Gastroenterol 2008, 42:527-532.

75. Toy M, Onder FO, Wormann T, Bozdayi AM, Schalm SW, Borsboom GJ, van Rosmalen J, Richardus JH, Yurdaydin C: Age- and region-specific hepatitis B prevalence in Turkey estimated using generalized linear mixed models: a systematic review. BMC Infect Dis 2011, 11:337.

76. Ferraro D, Genovese D, Argentini C, Giordano V, Pizzillo P, Stroffolini T, Craxi A, Rapicetta M, Di Stefano R: Phylogenetic reconstruction of HCV genotype 1b dissemination in a small city centre: the Camporeale model. J Med Virol 2008, 80:1723-1731.

77. Rossi C, Shrier I, Marshall L, Cnossen S, Schwartzman K, Klein MB, Schwarzer G, Greenaway C: Seroprevalence of chronic hepatitis B virus infection and prior immunity in immigrants and refugees: a systematic review and meta-analysis. PLoS One 2012, 7:e44611.

78. Anderson EM, Mandeville RP, Hutchinson SJ, Cameron SO, Mills PR, Fox R, Ahmed S, Taylor A, Spence E, Goldberg DJ: Evaluation of a general practice based hepatitis C virus screening intervention. Scott Med J 2009, 54:3-7.

79. European Monitoring Centre for Drugs and Drug Addiction (EMCDDA) Annual report 2012: the state of the drugs problem in Europe. Luxembourg: Publications Office of the European Union; 2012.

80. Wiessing L, Blystad H: EMCDDA publishes guidelines on testing for HIV, viral hepatitis and other infections in injecting drug users. Euro Surveill 2010, 15(48).

81. European Monitoring Centre for Drugs and Drug Addiction (EMCDDA): Guidelines for testing HIV, viral hepatitis and other infections in injecting drug users. Lisbon; 2010. http://www.emcdda.europa.eu/publications/manuals/ testing-guidelines.

82. Lee C, Gong Y, Brok J, Boxall EH, Gluud C: Effect of hepatitis B immunisation in newborn infants of mothers positive for hepatitis B surface antigen: systematic review and meta-analysis. BMJ 2006, 332:328-336.

83. World Health Organisation: Hepatitis B vaccines. Position paper. Weekly Epidemiol Record 2009, 40:405-420.

84. Van Der Veen YJ, Van Empelen P, Richardus JH: Development of a culturally tailored Internet intervention promoting hepatitis B screening in the Turkish community in the Netherlands. Health Promot Int 2012, 27:342-355.

85. Hahné S, Wormann Nee MT, Kretzschmar M: Migrants and hepatitis B: new strategies for secondary prevention needed. Eur J Publ Health 2009, 19:439.

86. Urbanus AT, van de Laar TJ, Vanden HA, Zuure FR, Speksnijder AG, Baaten GG, Heijman T, Vriend HJ, op de Coul EL, Coutinho RA, et al: Hepatitis C in the general population of various ethnic origins living in the Netherlands: Should non-Western migrants be screened? I Hepatol 2011, 55:1207-1214.
87. CDC: Recommendations for the identification of chronic hepatitis $\mathrm{c}$ virus infection among persons born during 1945-1965. MMWR Morb Mortal Wkly Rep 2012, 61:1-32

88. Martin NK, Vickerman P, Miners A, Foster GR, Hutchinson SJ, Goldberg DJ, Hickman M: Cost-effectiveness of hepatitis $C$ virus antiviral treatment for injection drug user populations. Hepatology 2012, 55:49-57.

89. Walker DG, Hutubessy R, Beutels P: WHO Guide for standardisation of economic evaluations of immunization programmes. Vaccine 2010, 28:2356-2359.

90. Beutels P, Edmunds WJ, Antoñanzas F, De Wit GA, Evans D, Feilden R, Fendrick AM, Ginsberg GM, Glick HA, Mast E, Péchevis M, Van Doorslaer EK van Hout BA: Viral Hepatitis Prevention Board: Economic evaluation of vaccination programmes: a consensus statement focusing on viral hepatitis. Pharmacoeconomics 2002, 20:1-7.

91. Quoilin S, Hutse V, Vandenberghe H, Claeys F, Verhaegen E, De Cock L, Van Loock F, Top G, Van Damme P, Vranckx R, et al: A population-based prevalence study of hepatitis $A, B$ and $C$ virus using oral fluid in Flanders, Belgium. Eur J Epidemiol 2007, 22:195-202.

92. Nemecek V, Castkova J, Fritz P, Linhartova A, Svandova E, Sramova H, Kriz B: The 2001 serological survey in the Czech Republic--viral hepatitis. Cent Eur J Public Health 2003, 11:S54-S61.

93. Nardone A, Anastassopoulou CG, Theeten H, Kriz B, Davidkin I, Thierfelder W, O'Flanagan D, Bruzzone B, Mossong J, Boot HJ, et al: A comparison of hepatitis B seroepidemiology in ten European countries. Epidemiol Infect 2009, 137:961-969.

94. Jilg W, Hottentrager B, Weinberger K, Schlottmann K, Frick E, Holstege A, Scholmerich J, Palitzsch KD: Prevalence of markers of hepatitis B in the adult German population. J Med Virol 2001, 63:96-102.

95. Thierfelder W, Hellenbrand W, Meisel H, Schreier E, Dortschy R: Prevalence of markers for hepatitis A, B and C in the German population. Results of the German National Health Interview and Examination Survey 1998. Eur J Epidemiol 2001, 17:429-435.

96. Gogos CA, Fouka KP, Nikiforidis G, Avgeridis K, Sakellaropoulos G, Bassaris H, Maniatis A, Skoutelis A: Prevalence of hepatitis B and C virus infection in the general population and selected groups in South-Western Greece. Eur J Epidemiol 2003, 18:551-557.

97. Raffaele A, Valenti M, lovenitti M, Matani A, Bruno ML, Altobelli E, D'Alessandro A, Barnabei R, Leonardis B, Taglieri G: High prevalence of HCV infection among the general population in a rural area of central Italy. Eur J Epidemiol 2001, 17:41-46.

98. Maio G, d'Argenio P, Stroffolini T, Bozza A, Sacco L, Tosti ME, Intorcia M, Fossi E, d'Alessio G, Kondili LA, et al: Hepatitis C virus infection and alanine transaminase levels in the general population: a survey in a southern Italian town. J Hepatol 2000, 33:116-120.

99. Pendino GM, Mariano A, Surace P, Caserta CA, Fiorillo MT, Amante A, Bruno S, Mangano C, Polito I, Amato F, et al: Prevalence and etiology of altered liver tests: a population-based survey in a Mediterranean town. Hepatology 2005, 41:1151-1159.

100. Coppola RC, Masia G, Pradat P, Trepo C, Carboni G, Argiolas F, Rizzetto M: Impact of hepatitis $C$ virus infection on healthy subjects on an Italian island. J Viral Hepat 2000, 7:130-137.

101. Di Stefano R, Stroffolini T, Ferraro D, Usticano A, Valenza LM, Montalbano L, Pomara G, Craxi A: Endemic hepatitis C virus infection in a Sicilian town: further evidence for iatrogenic transmission. J Med Virol 2002, 67:339-344

102. Dominguez A, Bruguera M, Vidal J, Plans P, Salleras L: Changes in the seroepidemiology of hepatitis B infection in Catalonia 1989-1996. Vaccine 2000, 18:2345-2350.

103. Salleras L, Dominguez A, Bruguera M, Plans P, Costa J, Cardenosa N, Batalla J, Plasencia A: Declining prevalence of hepatitis B virus infection in Catalonia (Spain) 12 years after the introduction of universal vaccination. Vaccine 2007, 25:8726-8731.

104. Hoffmann G, Berglund G, Elmstahl S, Eriksson S, Verbaan H, Widell A, Lindgren S: Prevalence and clinical spectrum of chronic viral hepatitis in a middle-aged Swedish general urban population. Scand J Gastroenterol 2000, 35:861-865.

105. Akcam FZ, Uskun E, Avsar K, Songur Y: Hepatitis B virus and hepatitis C virus seroprevalence in rural areas of the southwestern region of Turkey. Int J Infect Dis 2009, 13:274-284.

106. Demirturk N, Demirdal T, Toprak D, Altindis M, Aktepe OC: Hepatitis B and $C$ virus in West-Central Turkey: seroprevalence in healthy individuals 
admitted to a university hospital for routine health checks. Turk J Gastroenterol 2006, 17:267-272.

107. Guven R, Ozcebe H, Cakir B: Hepatitis B prevalence among workers in Turkey at low risk for hepatitis B exposure. East Mediterr Health J 2006, 12:749-757.

108. Yildirim B, Barut S, Bulut Y, Yenisehirli G, Ozdemir M, Cetin I, Etikan I, Akbas A, Atis O, Ozyurt $H$, et al: Seroprevalence of hepatitis $B$ and $C$ viruses in the province of Tokat in the Black Sea region of Turkey: A population-based study. [see comment]. Turk J Gastroenterol 2009, 20:27-30.

109. Degertekin H, Tuzcu A, Yalcin K: Horizontal transmission of HBV infection among students in Turkey. Publ Health 2000, 114:411-412.

110. Mehmet D, Meliksah E, Serif Y, Gunay S, Tuncer O, Zeynep S: Prevalence of hepatitis $B$ infection in the southeastern region of Turkey: comparison of risk factors for HBV infection in rural and urban areas. Jpn J Infect Dis 2005, 58:15-19.

111. Van Damme $P$, Thyssen A, Van Loock F: Epidemiology of hepatitis C in Belgium: present and future. Acta Gastroenterol Belg 2002, 65:78-79.

112. Atanasova MV, Haydouchka IA, Zlatev SP, Stoilova YD, lliev YT, Mateva NG: Prevalence of antibodies against hepatitis $C$ virus and hepatitis $B$ coinfection in healthy population in Bulgaria. A seroepidemiological study. Minerva Gastroenterol Dietol 2004, 50:89-96.

113. Pradat P, Caillat-Vallet E, Sahajian F, Bailly F, Excler G, Sepetjan M, Trepo C, Fabry J, members A: Prevalence of hepatitis $C$ infection among general practice patients in the Lyon area, France. Eur J Epidemio/ 2001, 17:47-51.

114. Goritsas C, Plerou I, Agaliotis S, Spinthaki R, Mimidis K, Velissaris D, Lazarou N, Labropoulou-Karatza C: HCV infection in the general population of a Greek island: prevalence and risk factors. Hepatogastroenterology 2000, 47:782-785

115. Campello C, Poli A, Dal MG, Besozzi-Valentini F: Seroprevalence, viremia and genotype distribution of hepatitis $C$ virus: a community-based population study in northern Italy. Infection 2002, 30:7-12.

116. Alberti A, Noventa F, Benvegnu L, Boccato S, Gatta A: Prevalence of liver disease in a population of asymptomatic persons with hepatitis $C$ virus infection.[summary for patients in Ann Intern Med. 2002 Dec 17;137 (12):I36; PMID: 12484740]. Ann Intern Med 2002, 137:961-964.

117. Petti S, Divizia M, Donia D, D' Arca AS, Tarsitani G, Fabi G, Pana A: Analysis of the shift of the transmission pattern for hepatitis $C$ in a community in Central Italy. New Microbiol 2006, 29:207-209.

118. Montella M, Crispo A, Grimaldi M, Angeletti C, Amore A, Ronga D, Sabbatini M, Pisani A, Spiteri D, Serraino D: Prevalence of hepatitis $C$ virus infection in different population groups in southern Italy.[erratum appears in Infection. 2005 Apr;33(2):103 Note: Sabbatici, M [corrected to Sabbatini, M]]. Infection 2005, 33:9-12.

119. Slavenburg S, Verduyn-Lunel FM, Hermsen JT, Melchers WJG, te Morsche RHM, Drenth JPH: Prevalence of hepatitis $C$ in the general population in the Netherlands. Neth J Med 2008, 66:13-17.

120. Bielawski K, Wlasiuk M, Truskolawska M, Falkiewicz B: HCV infection in Poland. Arch Med Res 2000, 31:532-535.

121. Gheorghe L, lacob S, Csiki IE: Prevalence of hepatitis C in Romania: different from European rates? J Hepatol 2008, 49:661-662

122. Dominguez A, Bruguera M, Vidal J, Plans P, Salleras L: Community-based seroepidemiological survey of HCV infection in Catalonia, Spain. J Med Virol 2001, 65:688-693.

123. Riestra S, Fernandez E, Leiva P, Garcia S, Ocio G, Rodrigo L: Prevalence of hepatitis $C$ virus infection in the general population of northern Spain. [see comment]. Eur J Gastroenterol Hepatol 2001, 13:477-481.

124. Balogun MA, Ramsay ME, Hesketh LM, Andrews N, Osborne KP, Gay NJ, Morgan-Capner P: The prevalence of hepatitis $C$ in England and Wales. J Infect 2002, 45:219-226.

125. Moher D, Liberati A, Tetzlaff J, Altman DG: Preferred reporting items for systematic reviews and meta-analyses: the PRISMA statement. PLoS Med 2009, 6:e1000097.

126. Altindis M, Yilmaz S, Dikengil T, Acemoglu H, Hosoglu S: Seroprevalence and genotyping of hepatitis $B$, hepatitis $C$ and HIV among healthy population and Turkish soldiers in Northern Cyprus. World J Gastroenterol 2006, 12:6792-6.

127. Offergeld R, Faensen D, Ritter S, Hamouda O: Human immunodeficiency virus, hepatitis $C$ and hepatitis $B$ infections among blood donors in Germany 2000-2002: risk of virus transmission and the impact of nucleic acid amplification testing. Euro Surveill 2005, 10:8-11.

128. Zervou EK, Dalekos GN, Boumba DS, Tsianos EV: Value of anti-HBc screening of blood donors for prevention of HBV infection: results of a 3-year prospective study in Northwestern Greece. Transfusion 2001, 41:652-8.
129. Manzini P, Girotto M, Borsotti R, Giachino O, Guaschino R, Lanteri M, et al: Italian blood donors with anti-HBc and occult hepatitis B virus infection. Haematologica 2007, 92:1664-70.

130. Kalibatas V: Payment for whole blood donations in Lithuania: the risk for infectious disease markers.[see comment]. Vox Sang 2008, 94:209-15.

131. Chlabicz S, Bonifatiuk I, Radziwon P: Prevalence of hepatitis C virus antibodies among blood donors in north-eastern Poland. Hepatol 2005, 33:206-10.

132. Eiras A, Sauleda S, Planelles D, Sedeno M, Ibarra A, Vesga MA, et al: HCV screening in blood donations using RT-PCR in mini-pool: the experience in Spain after routine use for 2 years. Transfusion 2003, 43:713-20.

133. Cowan SA, Bagdonaite J, Qureshi K: Universal hepatitis B screening of pregnant women in Denmark ascertains substantial additional infections: results from the first five months. Euro Surveill 2006, 11:E060608.

134. Denis F, Ranger-Rogez S, Alain S, Mounier M, Debrock C, Wagner A, et al: Screening of pregnant women for hepatitis B markers in a French Provincial University Hospital (Limoges) during 15 years. Eur J Epidemiol 2004, 19:973-8.

135. Knorr B, Maul H, Schnitzler P: Prevalence of hepatitis B virus infection among women at reproductive age at a German university hospital. J Clin Virol 2008, 42:422-4.

136. Papaevangelou V, Hadjichristodoulou C, Cassimos D, Theodoridou M: Adherence to the screening programme for HBV infection in pregnant women delivering in Greece. BMC Infect Dis 2006, 6:84

137. Healy CM, Cafferkey MT, Butler KM, Cahill I, McMorrow J, Philbin M, et al: Antenatal hepatitis B screening - is there a need for a national policy? Ir Med J 2001, 94:111-2.

138. Stroffolini T, Bianco E, Szklo A, Bernacchia R, Bove C, Colucci M, et al: Factors affecting the compliance of the antenatal hepatitis B screening programme in Italy. Vaccine 2003, 21:1246-9.

139. van Steenbergen JE, Leentvaar-Kuijpers A, Baayen D, Dukers HT, van Doornum GJ, van den Hoek JA, et al: Evaluation of the hepatitis B antenatal screening and neonatal immunization programme in Amsterdam, 1993-1998. Vaccine 2001, 12:7-11.

140. Stanekova D, Adamcakova J, Kopilcova T, Kotuliak J, Vaculikova E, Habekova M et al: Serological markers of selected sexually and blood transmitted infections in pregnant women and in newborns of HIV-positive mothers in the Slovak Republic. Cent Eur J Publ Health 2006, 14:104-8.

141. Salleras L, Dominguez A, Bruguera M, Plans P, Espunes J, Costa J, et al: Seroepidemiology of hepatitis $B$ virus infection in pregnant women in Catalonia (Spain). J Clin Virol 2009, 44:329-32.

142. Beckers K, Schaad UB, Heininger U: Compliance with antenatal screening for hepatitis B surface antigen carrier status in pregnant women and consecutive procedures in exposed newborns. Eur J Pediatr 2004, 163:654-7.

143. Anderson SR, Righarts A, Maguire H: Surveillance of antenatal infectionsHIV, hepatitis B, syphilis and rubella susceptibility in London. Commun Dis Publ Health 2004, 7:251-7.

144. Hillemanns P, Dannecker C, Kimmig R, Hasbargen U: Obstetric risks and vertical transmission of hepatitis $C$ virus infection in pregnancy. Acta Obstet Gynecol Scand 2000, 79:543-7.

145. Panagopoulos P, Economou A, Kasimi A, Spyropoulou P, Kanellopoulos N, Dadiotis $L$, et al: Prevalence of hepatitis $B$ and $C$ in the maternity department of a Greek district hospital. J Matern Fetal Neonatal Med 2004, 16:106-10.

146. Raptopoulou-Gigi M, Orphanou E, Lalla TH, Lita A, Garifallos A: Prevalence of hepatitis $C$ virus infection in a cohort of pregnant women in northern Greece and transmission of HCV from mother to child. Eur J Epidemiol 2001, 17:263-6.

147. Baldo V, Floreani A, Menegon T, Grella P, Paternoster DM, Trivello R: Hepatitis $C$ virus, hepatitis $B$ virus and human immunodeficiency virus infection in pregnant women in North-East Italy: a seroepidemiological study. Eur J Epidemiol 2000, 16:87-91.

148. Conte D, Fraquelli M, Prati D, Colucci A, Minola E: Prevalence and clinical course of chronic hepatitis $\mathrm{C}$ virus (HCV) infection and rate of HCV vertical transmission in a cohort of 15,250 pregnant women. Hepatology 2000, 31:751-5.

149. Veronesi L, Verrotti Di Pianella C, Benassi L, Benaglia G, Affanni P, Tanzi ML: Mother to child transmission of hepatitis $C$ virus in a province of northern Italy. J Prev Med Hyg 2007, 48:47-9. 
150. Prasad LR, Massery Spicher V, Kammerlander R, Zwahlen M: Hepatitis C in a sample of pregnant women in Switzerland: seroprevalence and sociodemographic factors. Swiss Med Wkly 2007, 137:27-32.

151. Ades AE, Parker S, Walker J, Cubitt WD, Jones R: HCV prevalence in pregnant women in the UK. Epidemiol Infect 2000, 125:399-405.

152. Balogun MA, Ramsay ME, Parry JV, Donovan L, Andrews NJ, Newham JA, et al: The prevalence and genetic diversity of hepatitis $C$ infection in antenatal clinic attenders in two regions of England. Epidemiol Infect 2000, 125:705-12.

153. Ward C, Tudor-Williams G, Cotzias T, Hargreaves S, Regan L, Foster GR: Prevalence of hepatitis $C$ among pregnant women attending an inner London obstetric department: uptake and acceptability of named antenatal testing.[see comment]. Gut 2000, 47:277-80

154. Goldberg D, Mclntyre PG, Smith R, Appleyard K, Dunlop J, Taylor A, et al: Hepatitis $C$ virus among high and low risk pregnant women in Dundee: unlinked anonymous testing. Br J Obstet Gyn 2001, 108:365-70.

155. Hutchinson SJ, Goldberg DJ, King M, Cameron SO, Shaw LE, Brown A, et al: Hepatitis $C$ virus among childbearing women in Scotland: prevalence, deprivation, and diagnosis. Gut 2004, 53:593-8.

156. Roussos A, Goritsas C, Pappas T, Spanaki M, Papadaki P, Ferti A: Prevalence of hepatitis B and C markers among refugees in Athens. World J Gastroenterol 2003, 9:993-5.

157. Chironna M, Germinario C, Lopalco PL, Quarto M, Barbuti S: HBV, HCV and HDV infections in Albanian refugees in Southern Italy (Apulia region). Epidemiol Infect 2000, 125:163-7.

158. Palumbo E, Scotto G, Faleo G, Cibelli DC, Angarano G: Prevalence of HBV genotypes in South American immigrants affected by HBV-related chronic active hepatitis. Braz I Infect Dis 2007, 11:311-3.

159. Palumbo E, Scotto G, Faleo G, Cibelli DC, Saracino A, Angarano G: Prevalence of HBV-genotypes in immigrants affected by HBV-related chronic active hepatitis. Arq Gastroenterol 2007, 44:54-7.

160. Majori S, Baldo V, Tommasi I, Malizia M, Floreani A, Monteiro G, et al: Hepatitis A, B, and C infection in a community of sub-Saharan immigrants living in Verona (Italy). J Travel Med 2008, 15:323-7.

161. Palumbo E, Scotto G, Cibelli DC, Faleo G, Saracin A, Angarano G: Immigration and hepatitis B virus: epidemiological, clinical and therapeutic aspects. East Mediterr Health J 2008, 14:784-90.

162. Manzardo C, Trevino B, Prat J, Cabezos J, Mongui E, Claveria I, et al: Communicable diseases in the immigrant population attended to in a tropical medicine unit: epidemiological aspects and public health issues. Travel Med Infect Dis 2008, 6:4-11.

163. Chironna M, Germinario C, Lopalco PL, Carrozzini F, Barbuti S, Quarto M: Prevalence rates of viral hepatitis infections in refugee Kurds from Iraq and Turkey.[see comment]. Infection 2003, 31:70-4.

164. Aweis D, Brabin B, Beeching N, Bunn J, Cooper C, Gardner K, et al: Hepatitis B prevalence and risk factors for $\mathrm{HBs} A g$ carriage amongst Somali households in Liverpool. Commun Dis Publ Health 2001, 4:247-52.

165. Michos A, Terzidis A, Kalampoki V, Pantelakis K, Spanos T, Petridou ET: Seroprevalence and risk factors for hepatitis $A, B$, and $C$ among Roma and non-Roma children in a deprived area of Athens, Greece. J Med Virol 2008, 80:791-7.

166. Chironna M, Germinario C, Lopalco PL, Carrozzini F, Quarto M: Prevalence of hepatitis virus infections in Kosovar refugees. Int I Infect Dis 2001, 5:209-13.

167. Veldhuijzen IK, van Driel HF, Vos D, de ZO, van Doornum GJ, de Man RA, et al: Viral hepatitis in a multi-ethnic neighborhood in the Netherlands: results of a community-based study in a low prevalence country. Int J Infect Dis 2009, 13:e9-e13.

168. Gyarmathy VA, Ujhelyi E, Neaigus A: HIV and selected blood-borne and sexually transmitted infections in a predominantly Roma (Gypsy) neighbourhood in Budapest, Hungary: a rapid assessment. Cent Eur J Publ Health 2008, 16:124-7.

169. Viviano E, Vitale F, Perna AM, Cataldo F, Firenze A, Romano N: Human herpesvirus 8 seroprevalence among internationally adopted children coming from Eastern Europe. New Microbiol 2009, 32:11-5.

\section{doi:10.1186/1471-2334-13-181}

Cite this article as: Hahné et al:: Infection with hepatitis B and C virus in Europe: a systematic review of prevalence and cost-effectiveness of screening. BMC Infectious Diseases 2013 13:181.

\section{Submit your next manuscript to BioMed Central and take full advantage of:}

- Convenient online submission

- Thorough peer review

- No space constraints or color figure charges

- Immediate publication on acceptance

- Inclusion in PubMed, CAS, Scopus and Google Scholar

- Research which is freely available for redistribution 